\title{
Formula Complexity of Ternary Majorities
}

\author{
Kenya Ueno \\ The Hakubi Center for Advanced Research and \\ Graduate School of Informatics, \\ Kyoto University \\ kenya@kuis.kyoto-u.ac.jp
}

\begin{abstract}
It is known that any self-dual Boolean function can be decomposed into compositions of 3-bit majority functions. In this paper, we define a notion of a ternary majority formula, which is a ternary tree composed of nodes labeled by 3-bit majority functions and leaves labeled by literals. We study their complexity in terms of formula size. In particular, we prove upper and lower bounds for ternary majority formula size of several Boolean functions. To devise a general method to prove the ternary majority formula size lower bounds, we give an upper bound for the largest separation between ternary majority formula size and DeMorgan formula size.
\end{abstract}

\section{Introduction}

The parity and majority functions are the most basic Boolean functions studied in the literature. When the number of input bits is odd, both of them are invariant under negations of all the input variables and the output (i.e., self-dual). The class of self-dual Boolean functions is closed under compositions. Therefore the recursive majority function defined by compositions of the 3-bit majority function is also self-dual.

A class of Boolean functions closed under compositions is called a Boolean clone. There are systematic studies on the relationship among Boolean clones known as Post's lattice [16]. (See also a survey [3] on Post's lattice with its applications.) According to the theory of Post's lattice, any monotone self-dual Boolean function can be decomposed into compositions of 3-bit majority functions. In other words, the 3-bit majority function is the universal gate for the class of monotone self-dual Boolean functions. On the other hand, the 3-bit Boolean function denoted by $\left(x_{1} \wedge \neg x_{2}\right) \vee\left(\neg x_{2} \wedge \neg x_{3}\right) \vee\left(\neg x_{3} \wedge x_{1}\right)$ is the universal gate for the class of self-dual Boolean functions. It is also representable by the 3-bit majority function with negations. Therefore any self-dual Boolean function can be also decomposed into compositions of 3-bit majority functions with negations.

Ibaraki and Kameda [8] developed a decomposition theory of monotone selfdual Boolean functions for the data structure called coteries which realize mutual exclusions in distributed systems. The theory was further investigated for selfdual Boolean functions in general by Bioch and Ibaraki [2], who gave the decom- 
position scheme of the 3-bit parity function into compositions of 3-bit majority functions. We will fully utilize this decomposition scheme in our results.

There are two kinds of formula models whose nodes are labeled by 2-bit Boolean functions, known as $\mathbf{U}_{2}$-formula (DeMorgan formula) and its extension $\mathbf{B}_{2}$-formula (full binary basis formula). Studies on formula complexity of $\mathbf{U}_{2^{-}}$ formulas and $\mathbf{B}_{2}$-formulas have a long period of history. Most of lower bound methods for $\mathbf{U}_{2}$-formula size are regarded as extensions of Khrapchenko [10] proving the $\Theta\left(n^{2}\right)$ matching bound for the parity function. However, there are some hard limitation against $\mathbf{U}_{2}$-formula complexity around $\Omega\left(n^{2}\right)$ revealed in $[7,9,11,12]$. In the case of $\mathbf{B}_{2}$-formula, the lower bound technique introduced by Nechiporuk [13] $\Omega\left(n^{2} / \log n\right)$ is the most classical and still the strongest method.

Independently from any choice of formula models, proving formula size lower bounds is one of the most important problems in computational complexity theory as a weaker version of the circuit size lower bound problem and $\mathbf{P} \neq \mathbf{N P}$. A super-polynomial formula size lower bound for a function in some complexity class (e.g., $\mathbf{N P}$ ) including $\mathbf{N C}^{1}$ implies a separation between the two complexity classes (e.g., $\mathbf{N C}^{1} \neq \mathbf{N P}$ ). The complexity class $\mathbf{N C}^{1}$ is defined in terms of logarithm circuit depth, which turns out to be equivalent to polynomial formula size. Therefore, the effect of the basis for formula complexity is also significant from the viewpoint of logical circuit design. With all the effort, it is extremely hard to give a slight improvement for the formula size problem even for a basic Boolean functions such as the majority function. Therefore there are fewer achievements in recent years concerned with formula complexity in spite of its importance.

In this paper, we consider a formula model $\mathbf{M A} \mathbf{A J}_{3}$-formula (ternary majority formula) besides $\mathbf{U}_{2}$-formula and $\mathbf{B}_{2}$-formula. Every node of a $\mathbf{M A} \mathbf{J}_{3}$-formula is labeled by the 3-bit majority function while every node of a $\mathbf{U}_{2}$-formula and $\mathbf{B}_{2}$ formula is labeled by a 2-bit Boolean function. We will prove the $\mathbf{M A} \mathbf{J}_{3}$-formula size lower and upper bounds in Section 4 and 5, respectively. To prove the lower bounds, we will show that the largest separation between $\mathbf{M A} \mathbf{J}_{3}$-formula and $\mathbf{U}_{2}$-formula complexity is at most $O\left(n^{\log _{2} 3}\right)$ in Section 3. It can be regarded as analogue of Pratt's result [17], which showed the largest separation between $\mathbf{B}_{2}$-formula complexity and $\mathbf{U}_{2}$-formula complexity is at most $O\left(n^{\log _{3} 10}\right)$.

Our work is intended as a basis towards further studies on $\mathbf{M A} \mathbf{J}_{3}$-formula and any similar kinds of circuits and formula models. Since $\mathbf{M A} \mathbf{J}_{3}$-formula can be seen as the most simplified form of threshold circuits as well as neural networks, there are possibilities to utilize related techniques. We hope that developing a new stream of studies on $\mathbf{M A} \mathbf{A} \mathbf{J}_{3}$-formulas will contribute a new progress revealing the complexity of itself as well as other existing formula models.

\section{Definitions}

In this section, we summarize definitions concerned with Boolean functions and formula size. We assume that the readers are familiar with the basics of these concepts together with the notations of $O, o, \Omega, \omega$ and $\Theta$. 


\subsection{Boolean Functions}

In this paper, we consider the following Boolean functions. Through the paper, $n$ means the number of input bits.

Definition 1 (Boolean Functions). The parity function $\mathbf{P A R}_{n}:\{0,1\}^{n} \mapsto$ $\{0,1\}$ is defined by

$$
\operatorname{PAR}_{n}\left(x_{1}, \cdots, x_{n}\right)=\left\{\begin{array}{lll}
1 & \left(\sum_{i=1}^{n} x_{i} \equiv 1\right. & \bmod 2), \\
0 & \left(\sum_{i=1}^{n} x_{i} \equiv 0\right. & \bmod 2) .
\end{array}\right.
$$

The majority function $\mathbf{M A} \mathbf{J}_{2 l+1}:\{0,1\}^{2 l+1} \mapsto\{0,1\}$ on odd number of input bits is defined by

$$
\mathbf{M A J}_{2 l+1}\left(x_{1}, \cdots, x_{n}\right)= \begin{cases}1 & \left(\sum_{i=1}^{n} x_{i} \geq l+1\right), \\ 0 & \left(\sum_{i=1}^{n} x_{i} \leq l\right)\end{cases}
$$

The recursive majority function $\mathbf{R e c M A J}_{3}^{h}:\{0,1\}^{3^{h}} \mapsto\{0,1\}$ is defined by

$$
\begin{aligned}
\operatorname{RecMAJ}_{3}^{h}\left(x_{1}, \cdots, x_{3^{h}}\right)=\operatorname{MAJ}_{3} & \operatorname{RecMAJ}_{3}^{h-1}\left(x_{1}, \cdots, x_{3^{h-1}}\right), \\
& \operatorname{RecMAJ}_{3}^{h-1}\left(x_{3^{h-1}+1}, \cdots, x_{2 \cdot 3^{h-1}}\right), \\
& \left.\operatorname{RecMAJ}_{3}^{h-1}\left(x_{2 \cdot 3^{h-1}+1}, \cdots, x_{3^{h}}\right)\right)
\end{aligned}
$$

with RecMAJ $_{3}^{1}=\mathbf{M A J}_{3}$.

We will define another Boolean function right before it will appear. The notions of monotone and self-dual for Boolean function are defined as follows.

Definition 2 (Monotone and Self-Dual). For Boolean vectors $\boldsymbol{x}=\left(x_{1}, \cdots, x_{n}\right)$ and $\boldsymbol{y}=\left(y_{1}, \cdots, y_{n}\right)$, we define $\boldsymbol{x} \leq \boldsymbol{y}$ if $x_{i} \leq y_{i}$ for all $i \in\{1, \cdots n\}$. A Boolean function $f$ is called monotone if $\boldsymbol{x} \leq \boldsymbol{y}$ implies $f(\boldsymbol{x}) \leq f(\boldsymbol{y})$ for any $\boldsymbol{x}, \boldsymbol{y} \in\{0,1\}^{n}$. A Boolean function $f$ is called self-dual if $f\left(x_{1}, \cdots, x_{n}\right)=$ $\neg f\left(\neg x_{1}, \cdots, \neg x_{n}\right)$ where $\neg$ denotes the negation, which flips 1 to 0 , and 0 to 1 .

\subsection{Formula Size}

In this paper, we consider the following three formula models. For each model, a literal means either a variable $x_{i}$ or the a negated variable $\neg x_{i}$ for some index $i$. Each formula is called monotone if it does not have negated variables. In the definition, the nodes $\wedge$ and $\vee$ mean the logical conjunction and disjunction, respectively. 
Definition 3 (Formula Models). A $\mathbf{U}_{2}$-formula is a binary tree with leaves labeled by literals and internal nodes labeled by $\wedge$ and $\vee . A \mathbf{B}_{2}$-formula is a binary tree with leaves labeled by literals and internal nodes labeled by any of 2-bit Boolean functions such as $\wedge, \vee$ and $\mathbf{P A R}_{2}$. A $\mathbf{M A} \mathbf{J}_{3}$-formula is a ternary tree with leaves labeled by literals and internal nodes labeled by $\mathbf{M A} \mathbf{J}_{3}$.

If we allow 0 and 1 in leaves along with literals, $\mathbf{M A} \mathbf{J}_{3}$-formulas can compute all the Boolean functions because $\mathbf{M} \mathbf{A} \mathbf{J}_{3}\left(x_{1}, x_{2}, 0\right)=x_{1} \wedge x_{2}$ and $\mathbf{M} \mathbf{A} \mathbf{J}_{3}\left(x_{1}, x_{2}, 1\right)=$ $x_{1} \vee x_{2}$. So the 3-bit majority function with 0 and 1 can be regarded as a kind of the universal gate for all the Boolean functions. In this sense, $\mathbf{M A} \mathbf{J}_{3}$-formula is yet another natural extension of $\mathbf{U}_{2}$-formula like $\mathbf{B}_{2}$-formula. Even if we do not allow 0 and 1 in leaves $\mathbf{M A} \mathbf{J}_{3}$-formulas can compute all the self-dual Boolean functions. Furthermore, even if we allow only variables without negations, they can compute all the monotone self-dual Boolean functions.

The formula size for each formula model is defined as follows. For the convenience, we will not distinguish a Boolean function $f$ and a formula computing $f$. Note that $L_{\mathbf{M A J}_{3}}(f)$ is defined only for self-dual Boolean functions while $L_{\mathbf{B}_{2}}(f)$ and $L_{\mathbf{U}_{2}}(f)$ are defined for all Boolean functions.

Definition 4 (Formula Size). The size of a formula is its number of leaves for any formula model. We define the formula size of a Boolean function $f$ as the size of the smallest formula computing $f$. We denote the size of $\mathbf{U}_{2}$-formula, $\mathbf{B}_{2}$-formula and $\mathbf{M A} \mathbf{J}_{3}$-formula of a Boolean function $f$ by $L_{\mathbf{B}_{2}}(f), L_{\mathbf{U}_{2}}(f)$ and $L_{\mathbf{M A J}_{3}}(f)$, respectively. We will sometimes abbreviate $L_{\mathbf{U}_{2}}(f)$ to $L(f)$ for simplicity.

\section{Translation from Ternary Majority Formulas to DeMorgan Formulas}

In this section, we analyze the relation between $\mathbf{M A} \mathbf{J}_{3}$-formula complexity and $\mathbf{U}_{2}$-formula complexity. The results in this section will be useful to derive a $\mathbf{M A J}_{3}$-formula size lower bound from a $\mathbf{U}_{2}$-formula size lower bound for the same function as shown in Section 4. We begin with the following simple proposition.

Proposition 1. $L_{\mathbf{M A J}_{3}}\left(\operatorname{RecMAJ}_{3}^{h}\right)=3^{h}$.

Proof. The upper bound $L_{\mathbf{M A J}_{3}}\left(\mathbf{R e c M A J}_{3}^{h}\right) \leq 3^{h}$ follows from the same construction as the definition. The lower bound $L_{\mathbf{M A J}_{3}}\left(\mathbf{R e c M A J}_{3}^{h}\right) \geq 3^{h}$ is also immediate because it depends on all the variables.

From a majority formula $L\left(\mathbf{M A J} \mathbf{J}_{3}\right) \leq 5$, we can recursively construct a formula for the recursive majority function whose size is $5^{h}$. Therefore we have an upper bound $L\left(\mathbf{R e c M A}_{3}^{h}\right) \leq 5^{h}$, i.e., $L\left(\mathbf{R e c M A J}_{3}^{h}\right) \in O\left(L_{\mathbf{M A J}_{3}}(f)^{1.4650}\right)$. Similarly, the best upper bound we know for $\mathbf{B}_{2}$-formula is also $L_{\mathbf{B}_{2}}\left(\mathbf{R e c M A} \mathbf{J}_{3}^{h}\right) \leq$ $5^{h}$. The quantum adversary bound [11], which is useful to prove $\mathbf{U}_{2}$-formula size lower bounds, has a nice composition property written as $\operatorname{ADV}(f \cdot g) \geq$ 
$\mathbf{A D V}(f) \cdot \mathbf{A D V}(g)$. It implies a formula size lower bound $4^{h} \leq L\left(\mathbf{R e c M A} \mathbf{J}_{3}^{h}\right)$, i.e. $L\left(\mathbf{R e c M A J}_{3}^{h}\right) \in \Omega\left(L_{\mathbf{M A J}_{3}}(f)^{1.2618}\right)$.

We call the value $\gamma$ an expansion factor from a $\mathbf{M A} \mathbf{J}_{3}$-formula into $\mathbf{U}_{2^{-}}$ formula for an arbitrary self-dual Boolean function $f$ if $L(f) \in O\left(\left(L_{\mathbf{M A J}_{3}}(f)\right)^{\gamma}\right)$. In the case of the recursive majority function, we can prove $\gamma \geq \log _{3} 5$ by solving $5 \cdot a^{\gamma} \leq(3 a)^{\gamma}$ where $L_{\mathbf{M A J}_{3}}\left(f_{1}\right)=L_{\mathbf{M A J}_{3}}\left(f_{2}\right)=L_{\mathbf{M A J}_{3}}\left(f_{3}\right)=a$. At first glance, the recursive majority function seems to have the largest expansion factor $\log _{3} 5$ from a $\mathbf{M} \mathbf{A} \mathbf{J}_{3}$-formula into a $\mathbf{U}_{2}$-formula among all the $\mathbf{M A} \mathbf{J}_{3}$-formulas. Surprisingly, this is not true as we prove in the next lemma.

Lemma 1. For any self-dual Boolean function $f$,

$$
L(f) \in O\left(L_{\mathbf{M A J}_{3}}(f)^{\log _{2} 3}\right) \subseteq O\left(L_{\mathbf{M A J}_{3}}(f)^{1.5850}\right) .
$$

Proof. We are looking for the largest formula expansion from a $\mathbf{M A} \mathbf{J}_{3}$-formula into a $\mathbf{U}_{2}$-formula. Differently from the recursive majority function, the same variable might appear more than once in a ternary majority formula for an arbitrary Boolean function $f$. In this case, the expanded $\mathbf{U}_{2}$-formula can shrink more. So we can concentrate on the case in which all the variable appear exactly once. That is, $L_{\mathbf{M A J}_{3}}(f)=n$.

We assume that $L(f) \leq \beta \cdot L_{\mathrm{MAJ}_{3}}(f)^{\gamma}$ for any self-dual Boolean function and consider an inductive argument. The expansion factor $\gamma$ must satisfy an inequality

$$
\begin{aligned}
L(f) & \leq 2 \cdot \beta \cdot\left(L_{\mathbf{M A J}_{3}}\left(f_{1}\right)\right)^{\gamma}+2 \cdot \beta \cdot\left(L_{\mathbf{M A J}_{3}}\left(f_{2}\right)\right)^{\gamma}+\beta \cdot\left(L_{\mathbf{M A J}_{3}}\left(f_{3}\right)\right)^{\gamma} \\
& \leq \beta \cdot\left(L_{\mathbf{M A J}_{3}}(f)\right)^{\gamma}
\end{aligned}
$$

by looking at a formula expansion from a $\mathbf{M} \mathbf{A} \mathbf{J}_{3}$-formula $f=\mathbf{M} \mathbf{A} \mathbf{J}_{3}\left(f_{1}, f_{2}, f_{3}\right)$ into a $\mathbf{U}_{2}$-formula $f=\left(f_{1} \wedge f_{2}\right) \vee\left(\left(f_{1} \vee f_{2}\right) \wedge f_{3}\right)$. This expansion is processed from leaves to the root in a recursive way.

We can assume that $L_{\mathbf{M A J}_{3}}\left(f_{1}\right) \leq L_{\mathbf{M A J}_{3}}\left(f_{2}\right) \leq L_{\mathbf{M A J}_{3}}\left(f_{3}\right)$ without loss of generality. We set $L_{\mathbf{M A J}_{3}}\left(f_{1}\right)=a-b, L_{\mathbf{M A J}_{3}}\left(f_{2}\right)=a+b$ and $L_{\mathbf{M A J}_{3}}\left(f_{3}\right)=a+c$ where $a>b \geq 0$ and $c \geq b \geq 0$. In this case, we need to find the minimum value of $\gamma$ which always satisfies

$$
2 \cdot(a-b)^{\gamma}+2 \cdot(a+b)^{\gamma}+(a+c)^{\gamma} \leq(3 a+c)^{\gamma} .
$$

So we set

$$
p(a, b, c, \gamma)=(3 a+c)^{\gamma}-(a+c)^{\gamma}-2 \cdot(a+b)^{\gamma}-2 \cdot(a-b)^{\gamma}
$$

and seek the minimum value of $\gamma$ such that $p(a, b, c, \gamma) \geq 0$ for any $a>b \geq 0$ and $c \geq b>0$.

First we fix $a, b$ and $\gamma$ and consider

$$
q(\alpha)=(3+\alpha)^{\gamma}-(1+\alpha)^{\gamma}
$$

where $\alpha=\frac{c}{a}(0<\alpha)$. By the derivative $y^{\prime}=\gamma \cdot x^{\gamma-1}$ of $y=x^{\gamma},(3+\alpha)^{\gamma}$ increases more than $(1+\alpha)^{\gamma}$ whenever $\alpha$ slightly increases. So $q(\alpha)$ monotonically increases 
as $\alpha$ increases. To minimize $p(a, b, c, \gamma)$ for fixed $\gamma$, we would like to minimize $q(\alpha)$ and had better $\alpha$ be as small as possible. Hence we set $c=b$ because $c \geq b$.

Next we consider

$$
r(\alpha, \gamma)=\frac{p(a, b, b, \gamma)}{a^{\gamma}}=(3+\alpha)^{\gamma}-3 \cdot(1+\alpha)^{\gamma}-2 \cdot(1-\alpha)^{\gamma}
$$

where $\alpha=\frac{b}{a}(0 \leq \alpha<1)$. Since $r(\alpha, \gamma) \geq 0$ for any $\alpha(0 \leq \alpha<1)$ implies $p(a, b, c, \gamma) \geq 0$ for any $a, b$ and $c$, it suffices to seek the minimum $\gamma$ which satisfies this condition.

It is easy to see that $\log _{3} 5$ is not the largest expansion factor because

$$
r\left(1, \log _{3} 5\right) \approx-0.660928<0
$$

while

$$
r\left(1, \log _{3} 5\right)=0 .
$$

On the other hand, $\log _{2} 3$ seems to be a good candidate which is very near to the largest expansion factor because

$$
r\left(0, \log _{2} 3\right) \approx 0.704522>0
$$

and

$$
r\left(1, \log _{2} 3\right) \approx 1.77636 \times 10^{-15}>0 .
$$

To confirm $r\left(0, \log _{2} 3\right) \geq 0$ for $0 \leq \alpha<1$, it is sufficient to draw the graph of $r\left(\alpha, \log _{2} 3\right)(0 \leq \alpha<1)$ as shown in Figure 1. (Strictly speaking, it requires a rigorous analysis on $r\left(\alpha, \log _{2} 3\right)$, but we omit it in this paper.)

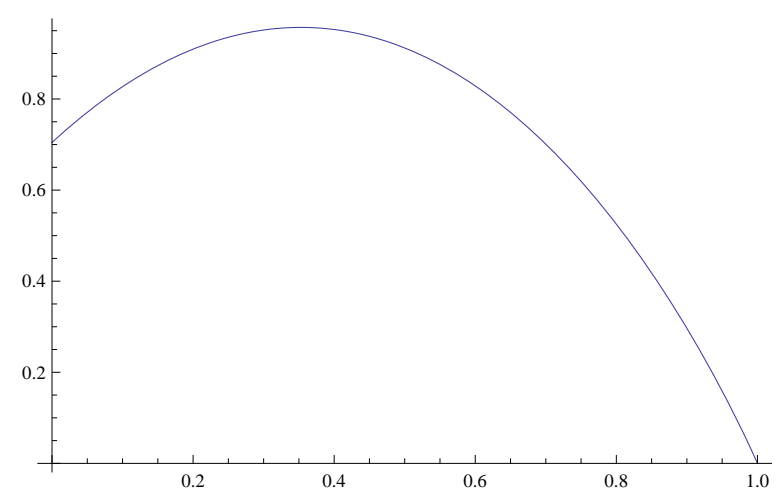

Fig. 1. $r\left(\alpha, \log _{2} 3\right)=(3+\alpha)^{\log _{2} 3}-3 \cdot(1+\alpha)^{\log _{2} 3}-2 \cdot(1-\alpha)^{\log _{2} 3}(0 \leq \alpha<1)$.

Therefore the largest expansion factor is at most $\log _{2} 3$, which is given for a $\mathbf{M A J} \mathbf{J}_{3}$-formula with $a-1=b=c$, i.e., $L_{\mathbf{M A J}_{3}}\left(f_{1}\right)=1$ and $L_{\mathbf{M A J}_{3}}\left(f_{2}\right)=$ $L_{\mathbf{M A J}_{3}}\left(f_{3}\right)$ for each subtree. 
Pratt [17] has proved

$$
L_{\mathbf{U}_{2}}(f) \in O\left(\left(L_{\mathbf{B}_{2}}(f)\right)^{\log _{3} 10}\right) \subseteq O\left(\left(L_{\mathbf{B}_{2}}(f)\right)^{2.096}\right) .
$$

The exponent $\log _{3} 10$ is derived from the $\mathbf{U}_{2}$-formula size of 10 for the 3 -bit parity function. The above lemma can be seen as an analogue of Pratt's bound [17] for the relation between $\mathbf{M A} \mathbf{J}_{3}$-formulas and $\mathbf{U}_{2}$-formulas.

\section{Ternary Majority Formula Size Lower Bounds}

In this section, we devise a general method to prove ternary majority formula size lower bounds. In general, we can derive a $\mathbf{M A} \mathbf{J}_{3}$-formula size lower bound for an arbitrary Boolean function from a $\mathbf{U}_{2}$-formula size lower bound of the same function using Lemma 1 as follows.

Theorem 1. For any self-dual Boolean function $f$ such that $L(f) \in \Omega\left(n^{c}\right)$, $L_{\mathrm{MAJ}_{3}}(f) \in \Omega\left(n^{c / \log _{2} 3}\right)$.

Proof. By Lemma 1, an upper bound for $\mathbf{U}_{2}$-formula size expanded from a $\mathbf{M A J}_{3}$-formula of size $N$ is at most $O\left(N^{\log _{2} 3}\right)$. This size must be not smaller than the formula size lower bound $L(f) \in \Omega\left(n^{c}\right)$. Therefore we have obtained the theorem.

From $\mathbf{U}_{2}$-formula size lower bounds of $L\left(\mathbf{P A R} \mathbf{R}_{n}\right) \in \Omega\left(n^{2}\right)$ and $L\left(\mathbf{M A} \mathbf{J}_{n}\right) \in$ $\Omega\left(n^{2}\right)$ by Khrapchenko [10], we have the following corollaries. After completion of our work, we have noticed that the lower bound for the parity function is weaker than 1.33 of Chokler and Zwick [4] using the random restriction technique. Still, our lower bound method has merit in the sense that it can be applied for any Boolean function.

Corollary 1. For any $n=2 l+1$, we have $L_{\mathbf{M A J}_{3}}\left(\mathbf{P A R}_{2 l+1}\right) \in \Omega\left(n^{1.2618}\right)$ and $L_{\mathbf{M A J}_{3}}\left(\mathbf{M A J}_{2 l+1}\right) \in \Omega\left(n^{1.2618}\right)$.

Since $2 / \log _{2} 3=\log _{3} 4$, these lower bounds are equal to the $\mathbf{U}_{2}$-formula size lower bound for the recursive majority function accidentally. It seems to be difficult to give a matching $\mathbf{M A} \mathbf{J}_{3}$-formula size upper and lower bounds even for the parity function while we can obtain those for $\mathbf{U}_{2}$-formula and $\mathbf{B}_{2}$-formula. Both of them seem to be not tight and have room for further improvements.

The current best $\mathbf{B}_{2}$-formula size lower bound is $\Omega\left(n^{2} / \log n\right)$ shown by Nechiporuk [13] for the element distinctness function. We should note that Pratt's bound [17] $L_{\mathbf{U}_{2}}(f) \in O\left(\left(L_{\mathbf{B}_{2}}(f)\right)^{2.096}\right)$ is not sufficient to give a substantial lower bound larger than $n$ differently from the case of $\mathbf{M A} \mathbf{J}_{3}$-formula. This is because $\mathbf{U}_{2}$-formula size lower bounds have got stuck the barrier around $\Omega\left(n^{2}\right)[7,9,11,12]$ for almost all explicitly defined Boolean functions except the Andreev function discussed from now on.

The current best $\mathbf{U}_{2}$-formula size lower bound is $\Omega\left(n^{3-o(1)}\right)$ by Håstad [6] for the Andreev function [1]. We can define the Andreev function so that it is self-dual. 
Definition 5. The Andreev function $\mathbf{A}_{2 n}$ is composed of $2 n$ input bits which are divided into 2 parts. The first part consists of $n$ bits represent the truth table of a Boolean function $f$ on $\log n$ bits. The second part consists of $n$ bits which are also divided into $\log n$ blocks of $n / \log n$ bits. First, the function computes $\log n$ parity function on $n / \log n$ input bits from the second part of the input bits. and obtain $\log n$ output bits. Here we assume that the number of input bits for all the parity function is even. That is, $n / \log n$ is even. Then it computes the Boolean function $f$ represented by the first part with the obtained $\log n$ output bits as input bits.

With the slightly modification of the Andreev function from its original definition version, we can confirm that it is self-dual as follows.

Lemma 2. The Andreev function $\mathbf{A}_{2 n}$ defined as above is self-dual.

Proof. We consider the situation in which we flip all the input bits for the Andreev function. Because we defined the Andreev function so that the number of input bits for all the parity functions inside the Andreev function is even, the output bits are invariant when we flip all the input bits. In other words, the parity function with even number of input bits is anti-dual, i.e.,

$$
\mathbf{P A R}_{n / \log n}\left(x_{1}, \cdots, x_{n / \log n}\right)=\mathbf{P A R}_{n / \log n}\left(\neg x_{1}, \cdots, \neg x_{n / \log n}\right) .
$$

Therefore the Andreev function outputs a bit in the same position in the first part of the input bits after flipping all the input bits. On the other hand, this output bit has been also flipped. Hence the output bit of the Andreev function is also flipped after we flip all the input bits.

So our $\mathbf{M A J}_{3}$-formula size lower bound for the Andreev function is given as follows.

Theorem 2. $L_{\mathrm{MAJ}_{3}}\left(\mathbf{A}_{2 n}\right) \in \Omega\left(n^{1.8927}\right)$

Proof. Since we have defined the Andreev function so that it is self-dual, it can be represented by a $\mathbf{M A} \mathbf{J}_{3}$-formula from the theory of Post [16]. Moreover, the modification of the Andreev function does not affect the $\mathbf{U}_{2}$-formula size lower bound of $\Omega\left(n^{3-o(1)}\right)$ by Håstad [6]. Thus we can apply Theorem 1 and obtain the lower bound by $3 / \log _{2} 3 \approx 1.89279$.

\section{Ternary Majority Formula Size Upper Bounds}

In this section, we prove $\mathbf{M A J}_{3}$-formula size upper bounds of the parity and majority function. In both cases, the upper bounds are shown by utilizing the decomposition scheme of Bioch and Ibaraki [2] for the 3-bit parity function as

$$
\operatorname{PAR}_{3}\left(x_{1}, x_{2}, x_{3}\right)=[1,[\overline{1}, \overline{2}, \overline{3}],[\overline{1}, 2,3]]
$$

where we use notations $[i, j, k]=\mathbf{M A J}_{3}\left(x_{i}, x_{j}, x_{k}\right), i=x_{i}$ and $\bar{i}=\neg x_{i}$. From the decomposition scheme, we obtain $L_{\mathbf{M A J}_{3}}\left(\mathbf{P A R}_{3}\right) \leq 7$. We show that $\mathbf{M A} \mathbf{J}_{3^{-}}$ formula complexity is intermediate between $\mathbf{B}_{2}$-formula complexity and $\mathbf{U}_{2}$ complexity for both functions. 


\subsection{The Parity Function}

In the case of $\mathbf{U}_{2}$-formula, we can construct a 2-bit parity formula $\left(x_{1} \wedge \neg x_{2}\right) \vee$ $\left(\neg x_{1} \wedge x_{2}\right)$. By a recursive construction, we can prove an upper bound $L\left(\mathbf{P A R} \mathbf{R}_{n}\right) \leq$ $n^{2}$ where $n=2^{h}$ from a recursive inequality $L\left(\mathbf{P A R}_{2 n}\right) \leq 4 \cdot L\left(\mathbf{P A R} \mathbf{R}_{n}\right)$.

In the case of $\mathbf{M A} \mathbf{J}_{3}$-formula, we can decompose the $3^{h}$-bits parity function into a composition of a 3 -bit parity function and three $3^{h-1}$-bits parity functions. Thus we have a recursive inequality $L_{\mathbf{M A J}_{3}}\left(\mathbf{P A R}_{3^{h}}\right) \leq 7 \cdot L_{\mathbf{M A J}_{3}}\left(\mathbf{P A R}_{3^{h-1}}\right)$ from the decomposition scheme of the 3-bit parity function. Solving this inequality straightforwardly, we can show an upper bound $L_{\mathbf{M A J}_{3}}\left(\mathbf{P A R}_{3^{h}}\right) \in$ $O\left(n^{\log _{3} 7}\right) \subseteq O\left(n^{1.7712}\right)$. Actually we can give a better upper bound as follows.

Theorem 3 (See also [4]). $L_{\mathbf{M A J}_{3}}\left(\mathbf{P A R}_{2 l+1}\right) \in O\left(n^{1.7329}\right)$ where $n=2 l+1$.

Proof. For some constant $\alpha$, we consider decomposition of the $(2 \alpha+1) \cdot m$-bit parity function into a composition of a 3 -bit parity function with a $m$-bit parity function and two $\alpha \cdot m$-bit parity functions as follows.

$$
\begin{aligned}
\mathbf{P A R}_{(2 \alpha+1) \cdot m}\left(x_{1}, \cdots, x_{(2 \alpha+1) \cdot m}\right)=\mathbf{P A R}_{3}\left(\mathbf{P A R}_{m}\left(x_{1}, \cdots, x_{m}\right),\right. \\
\\
\mathbf{P A R}_{\alpha \cdot m}\left(x_{m+1}, \cdots, x_{(\alpha+1) \cdot m}\right), \\
\left.\mathbf{P A R}_{\alpha \cdot m}\left(x_{(\alpha+1) \cdot m+1}, \cdots, x_{(2 \alpha+1) \cdot m}\right)\right) .
\end{aligned}
$$

Here we can assume that $\alpha \cdot m$ is an odd integer by increasing or decreasing it at most 1 . In this case, $(2 \alpha+1) \cdot m$ becomes also an odd integer if $m$ is odd.

Let $S(n)=L_{\mathrm{MAJ}_{3}}\left(\mathbf{P A R}_{n}\right)$ and assume $S(n) \leq \beta \cdot n^{\gamma}$ for some constants $\beta, \gamma>0$ for any odd number $n$. By increasing the value of $\beta$, the slight modification which makes $\alpha \cdot m$ be an odd integer can be ignored for the following estimation of $\gamma$. By using decomposition scheme of the 3-bit parity function,

$$
\begin{aligned}
S((1+2 \alpha) \cdot m) & \leq 3 \cdot S(m)+2 \cdot S(\alpha \cdot m)+2 \cdot S(\alpha \cdot m) \\
& \leq\left(3+4 \cdot \alpha^{\gamma}\right) \cdot \beta \cdot n^{\gamma} .
\end{aligned}
$$

It suffices to show that the last expression is bounded by $(1+2 \alpha)^{\gamma} \cdot \beta \cdot n^{\gamma}$. Therefore we consider the minimum value of $\gamma$ which satisfies

$$
3+4 \cdot \alpha^{\gamma} \leq(1+2 \alpha)^{\gamma}
$$

by eliminating $\beta \cdot m^{\gamma}$ from both sides. We can verify that this inequality is satisfied when $\alpha=1.73896$ and $\gamma=1.73282$.

We have possibilities to improve the upper bound by analysis of the parity function with larger number of input bits. In this case, the proof will become much more complicated as the number of input bits increases. For example, we can construct $\mathbf{M A} \mathbf{J}_{3}$-formula of size 21 for the 5-bit parity function as

$$
\begin{array}{r}
\operatorname{PAR}_{5}\left(x_{1}, x_{2}, x_{3}, x_{4}, x_{5}\right)= \\
{[1,[\overline{1}, \overline{2},[\overline{3},[3,4,5],[3, \overline{4}, \overline{5}]]],[\overline{1}, 2,[\overline{1}, 2,[3,[\overline{3}, \overline{4}, \overline{5}],[\overline{3}, 4,5]]]]] .}
\end{array}
$$

From the construction, we can obtain the upper bound of $O\left(n^{\log _{5} 21}\right)=O\left(n^{1.8917}\right)$. This is far from the above upper bound. To obtain a better bound, we need much more succinct construction of a $\mathbf{M A} \mathbf{J}_{3}$-formula for the 5-bit parity function of size as close to $5^{1.7328}=16.262 \ldots$ as possible. 


\subsection{The Majority Function}

Our $\mathbf{M A} \mathbf{J}_{3}$-formula size upper bound for the majority function essentially relies on the general theory established by Paterson, Pippenger and Zwick [14]. Their idea is based on construction of a carry save adder from a full adder of fixed size as building blocks. Here we consider a full adder $\mathbf{F} \mathbf{A}_{3}$ from 3 bits to 2 bits. The first and second output bits $y_{1}, y_{2}$ of $\mathbf{F A}_{3}$ are the 3-bit parity and majority function, respectively.

In the case of $\mathbf{U}_{2}$-formula, the full adder $\mathbf{F} \mathbf{A}_{3}$ can be constructed by

$$
y_{1}=\left(x_{1} \wedge\left(\left(\neg x_{2} \vee x_{3}\right) \wedge\left(x_{2} \vee \neg x_{3}\right)\right)\right) \vee\left(\neg x_{1} \wedge\left(\left(x_{2} \wedge \neg x_{3}\right) \vee\left(\neg x_{2} \wedge x_{3}\right)\right)\right),
$$

and

$$
y_{2}=\left(x_{1} \wedge x_{2}\right) \vee\left(\left(x_{1} \vee x_{2}\right) \wedge x_{3}\right) .
$$

They defined the notion of the occurrence matrix. It summarizes the information of the number of occurrence in the formula. For example, the occurrence matrix of the above case is $M=\left(\begin{array}{lll}2 & 4 & 4 \\ 1 & 2 & 2\end{array}\right)$. In the first and second row of the matrix, each entry counts the number of occurrence of each variable in the first and second formula, respectively.

From the construction of an arbitrary fixed size full adder and its corresponding occurrence matrix, Paterson, Pippenger and Zwick [14] gave the following general upper bound method.

Theorem 4 ([14]). Let $M$ be an occurrence matrix of some full adder for some fixed basis and some Boolean function $f$. Let $\epsilon(M)$ be the maximum value of $\frac{1}{\gamma}$ such that $\|\boldsymbol{x}\|_{\gamma} \leq\|M \cdot \boldsymbol{x}\|_{\gamma}$ for any vector $\boldsymbol{x} \geq \mathbf{0}$ where $\|x\|_{\gamma}=\left(\sum_{i}\left|x_{i}\right|^{\gamma}\right)^{1 / \gamma}$. Then $O\left(n^{\epsilon(M)+o(1)}\right)$ gives a formula size upper bound for $f$ on the fixed basis.

By the theorem, we can derive a $\mathbf{U}_{2}$-formula size upper bound of $O\left(n^{4.70}\right)$. Paterson and Zwick [15] gave a construction of the full adder from 11 bits to 4 bits and an improved upper bound of $O\left(n^{4.57}\right)$.

In the case of $\mathbf{B}_{2}$-formula, Paterson, Pippenger and Zwick [14] proved a $\mathbf{B}_{2^{-}}$ formula size upper bound of $O\left(n^{3.21}\right)$ improved to $O\left(n^{3.13}\right)$ by Paterson and Zwick [15].

In the case of $\mathbf{M A} \mathbf{J}_{3}$-formula, the full adder $\mathbf{F A}_{3}$ can be constructed by $y_{1}=$ $[1,[\overline{1}, \overline{2}, \overline{3}],[\overline{1}, 2,3]]$ and $y_{2}=[1,2,3]$. So the corresponding occurrence matrix is $M=\left(\begin{array}{lll}3 & 2 & 2 \\ 1 & 1 & 1\end{array}\right)$. From this, we can obtain the following $\mathbf{M A} \mathbf{J}_{3}$-formula size upper bound for the majority function.

Theorem 5. $L_{\mathbf{M A J}_{3}}\left(\mathbf{M A J} \mathbf{J}_{2 l+1}\right) \in O\left(n^{3.7925}\right)$ where $n=2 l+1$.

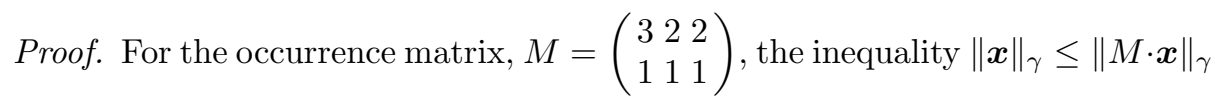
which appears in the theorem of Paterson, Pippenger and Zwick [14] can be interpreted as

$$
p(a, b, c, \gamma)=(3 \cdot a+2 \cdot b+2 \cdot c)^{\gamma}+(a+b+c)^{\gamma}-a^{\gamma}-b^{\gamma}-c^{\gamma} \geq 0 .
$$


If $L_{\mathbf{M A J}_{3}}\left(\mathbf{M A J}_{n}\right) \in O\left(n^{\gamma}\right)$, there exists $a, b, c>0$ such that $p(a, b, c, \gamma)<0$. We set $a=0.729608, b=c=1$ and $1 / \gamma=3.7925$. Then, we have

$$
p(a, b, c, \gamma) \approx-0.0000256657<0 .
$$

This certifies that the maximum value of $1 / \gamma$ which satisfies $\|\boldsymbol{x}\|_{\gamma} \leq\|M \cdot \boldsymbol{x}\|_{\gamma}$ for any vectors $\boldsymbol{x} \geq \mathbf{0}$ is less than 3.7925. (The optimality of the value $\gamma$ can be confirmed by numerical analysis. That is, the minimum value of $p(a, b, c, \gamma)>0$ for $\gamma=3.7924$.) Thus we have obtained the upper bound.

The best monotone $\mathbf{U}_{2}$-formula size upper bound for the majority function is $O\left(n^{5.3}\right)$ by a probabilistic construction of Valiant [18]. Following the analysis of Valiant's construction replaced by balanced compositions of the 3-bit majority function with random variables, we can construct a monotone $\mathbf{M A} \mathbf{J}_{3^{-}}$ formula whose size is $O\left(n^{4.2945}\right)\left(\supseteq O\left(n^{\log _{3 / 2} 3+\log _{2} 3}\right)\right)$. The size of its conversion into a monotone $\mathbf{U}_{2}$-formula is $O\left(n^{6.2913}\right)\left(\supseteq O\left(n^{\log _{3 / 2} 5+\log _{2} 5}\right)\right)$ and larger than Valiant's bound.

\section{Concluding Remarks}

In this paper, we have introduced the notion of $\mathbf{M A} \mathbf{J}_{3}$-formula and have shown the upper and lower bounds for $\mathbf{M A J}_{3}$-formula size of several Boolean functions. The results shown in this paper are summarized in Figure 2. The figure also shows comparison with $\mathbf{U}_{2}$-formula complexity and $\mathbf{B}_{2}$-formula complexity.

\begin{tabular}{|c||c|c|c|}
\hline & $\mathbf{B}_{2}$-formula & $\mathbf{M A J}_{3}$-formula & $\mathbf{U}_{2}$-formula \\
\hline \hline \multirow{2}{*}{ Parity } & $\Theta(n)$ & $O\left(n^{1.7329}\right)[4]$ & \multirow{2}{*}{$\Theta\left(n^{2}\right)[10]$} \\
\cline { 3 - 4 } & & $\Omega\left(n^{1.2618}\right), O\left(n^{1.3333}\right)[4]$ & \\
\hline \multirow{2}{*}{ Majority } & $O\left(n^{3.13}\right)[14,15]$ & $O\left(n^{3.7925}\right)$ & $O\left(n^{4.57}\right)[14,15]$ \\
\cline { 2 - 4 } & $\Omega(n \log n)[5]$ & $\Omega\left(n^{1.2618}\right)$ & $\Omega\left(n^{2}\right)[10]$ \\
\hline Recursive Majority & & $\Theta(n)$ & $O\left(n^{1.4650}\right)[11]$ \\
\cline { 3 - 4 } & & & $\Omega\left(n^{1.2618}\right)[11]$ \\
\hline Andreev & & $\Omega\left(n^{1.8927}\right)$ & $\Theta\left(n^{3-o(1)}\right)[6]$ \\
\hline
\end{tabular}

Fig. 2. Formula Size Upper and Lower Bounds

There are still large gaps between the upper and lower bounds even for the parity function while we have its matching bounds for $\mathbf{U}_{2}$-formula and $\mathbf{B}_{2}$ formula. The obvious open questions are how to close these gaps. We hope that a new technical discovery to clarify $\mathbf{M A} \mathbf{J}_{3}$-formula complexity will also shed light on resolving the stiff barrier against formula complexity of the existing models. 


\section{Acknowledgment}

This research is supported by the Kyoto University Hakubi Project and Grantsin-Aid for Scientific Research from the Japan Society for the Promotion of Science.

\section{References}

1. A. E. Andreev. On a method for obtaining more than quadratic effective lower bounds for the complexity of $\pi$-scheme. Moscow University Mathematics Bulletin, 42(1):63-66, 1987.

2. J. C. Bioch and T. Ibaraki. Decompositions of positive self-dual boolean functions. Discrete Mathematics, 140(1-3):23-46, 1995.

3. E. Böhler, N. Creignou, S. Reith, and H. Vollmer. Playing with boolean blocks, part I: Post's lattice with applications to complexity theory. ACM SIGACT News, 34(4):38-52, 2003.

4. H. Chockler and U. Zwick. Which bases admit non-trivial shrinkage of formulae? Computational Complexity, 10(1):28-40, 2001.

5. M. J. Fischer, A. R. Meyer, and M. S. Paterson. $\Omega(n \log n)$ lower bounds on length of Boolean formulas. SIAM Journal on Computing, 11(3):416-427, Aug. 1982.

6. J. Håstad. The shrinkage exponent of De Morgan formulas is 2. SIAM Journal on Computing, 27(1):48-64, Feb. 1998.

7. P. Hrubeš, S. Jukna, A. Kulikov, and P. Pudlák. On convex complexity measures. Theoretical Computer Science, 411:1842-1854, 2010.

8. T. Ibaraki and T. Kameda. A theory of coteries: Mutual exclusion in distributed systems. IEEE Transactions on Parallel and Distributed Computing, PDS-4(7):779-794, July 1993.

9. M. Karchmer, E. Kushilevitz, and N. Nisan. Fractional covers and communication complexity. SIAM Journal on Discrete Mathematics, 8(1):76-92, Feb. 1995.

10. V. M. Khrapchenko. Complexity of the realization of a linear function in the case of $\pi$-circuits. Mathematical Notes, 9:21-23, 1971.

11. S. Laplante, T. Lee, and M. Szegedy. The quantum adversary method and classical formula size lower bounds. Computational Complexity, 15(2):163-196, 2006.

12. T. Lee. A new rank technique for formula size lower bounds. In Proceedings of the 24th Annual Symposium on Theoretical Aspects of Computer Science (STACS 2007), Lecture Notes in Computer Science 4393, pages 145-156. Springer, 2007.

13. E. I. Neciporuk. A boolean function. DOKLADY: Russian Academy of Sciences Doklady. Mathematics (formerly Soviet Mathematics-Doklady), 7:999-1000, 1966.

14. M. S. Paterson, N. Pippenger, and U. Zwick. Optimal carry save networks. In Boolean function complexity, volume 169 of London Mathematical Society Lecture Note Series, pages 174-201. Cambridge University Press, 1992.

15. M. S. Paterson and U. Zwick. Shallow circuits and concise formulae for multiple addition and multiplication. Computational Complexity, 3(3):262-291, 1993.

16. E. L. Post. The two-valued iterative systems of mathematical logic, volume 5 of Annals Mathematical Studies. Princeton University Press, 1941.

17. V. R. Pratt. The effect of basis on size of Boolean expressions. In Proceedings of the 16th Annual Symposium on Foundations of Computer Science (FOCS 1975), pages 119-121. IEEE, 13-15 Oct. 1975.

18. L. G. Valiant. Short monotone formulae for the majority function. Journal of Algorithms, 5(3):363-366, Sept. 1984. 